\title{
Necesidades, Creencias y Prácticas en Salud Oral de Padres y Cuidadores de Preescolares: Un Enfoque Cualitativo
}

\author{
Needs, Beliefs and Practices in Oral Health of Preschoolers' \\ Parents and Caregivers: A Qualitative Scope
}

Paola Olave-Müller'; Valentina Fajreldin²; Lorena Coronado Vigueras ${ }^{3}$; Natalia López-Contreras ${ }^{4} \&$ María Teresa Valenzuela ${ }^{5}$

OLAVE-MÜLLER, P.; FAJRELDIN, V.; CORONADO, V. L.; LÓPEZ-CONTRERAS, N.; VALENZUELA, M. T. Necesidades, creencias y prácticas en salud oral de padres y cuidadores de preescolares: Un enfoque cualitativo. Int. J. Odontostomat., 15(4):888-897, 2021.

RESUMEN: A casi diez años de la implementación del exitoso programa público de promoción de la salud bucal para preescolares en Chile, se presenta el estudio previo que recogió la visión y significados para los padres acerca de temas de salud oral de sus hijos preescolares en un Chile urbano y rural de siete comunas distintas. Bien sabemos que la alta prevalencia de la caries dental en Chile y sus consecuencias a lo largo del ciclo vital, ha relevado la Promoción de Salud como una estrategia necesaria para la situación sanitaria de hace una década y para la actual a través de la implementación de programas con enfoque preventivo, como lo es el programa Sembrando Sonrisas, cuyo componente educativo se centra en los/as cuidadores de preescolares. En esta línea, cabe destacar que el Modelo de Creencias en Salud (MCS) actúa como un predictor para las prácticas en salud que desarrollan los/as cuidadores respecto a sus hijos, las cuales pueden ser activadas y/o modificadas por estrategias educativas o consejería otorgada por profesionales de la salud. Este estudio buscó explorar, mediante metodología cualitativa, los sistemas de creencias en torno a las necesidades en salud oral percibidas por padres y cuidadores/as de niños/ as preescolares que participaron del pilotaje del Programa preventivo de salud bucal en población preescolar el año 2007. Los resultados obtenidos evidencian elementos que pudieron condicionar la conducta en torno a los cuidados de la salud oral de las/ os cuidadores hacia los preescolares. Entre los hallazgos, se encuentra que los padres y apoderados atribuían los cuidados en salud oral a terceras personas (odontólogos y educadoras de párvulos), al tiempo que relatan una falta de información en términos comprensibles para que ellos puedan replicar prácticas adecuadas en sus hijos. Por otro lado, la postergación de la visita al odontólogo se explica debido a malas experiencias previas y falta de acceso a servicios de atención odontológica. Sin embargo, la salud dental es relevada desde un punto de vista estético y como un elemento importante en la superación de estigmas sociales.

PALABRAS CLAVE: promoción en salud oral, conductas en salud, salud oral.

INTRODUCCIÓN

La caries dental se ha establecido como la enfermedad más prevalente en la historia de la morbilidad oral en el mundo. De acuerdo a datos entregados por la Organización Mundial de la Salud (OMS) la prevalencia de caries a nivel mundial alcanza al $60 \%-90 \%$ de los escolares, aumentando casi al $100 \%$ durante la edad adulta (Fresno et al., 2019; Organización Mundial de la
Salud, 2020). La situación en Chile no dista mucho de éstas cifras, pues de acuerdo a estadísticas del Ministerio de Salud (MINSAL), el 17,52 \% de los niños de 2 años y la mitad de los párvulos de 4 años tienen historia de caries, agravados estos índices por determinantes como el nivel socioeconómico, mostrando que los párvulos de nivel socio económico bajo tiene aproximada-

\footnotetext{
${ }^{1}$ Facultad de Medicina, Departamento de Salud Pública, Universidad de La Frontera, Temuco, Chile.

${ }^{2}$ Facultad de Odontología, Universidad de Chile, Santiago, Chile.

${ }^{3}$ Universitat Pompeu Fabra, Barcelona, España.

${ }^{4}$ Universidad de La Frontera, Temuco, Chile.

${ }^{5}$ Universidad de Chile, Escuela de Salud Pública, Facultad de Medicina, Santiago, Chile.

Fuente de Financiamiento: Ministerio de Salud, Chile.
} 
OLAVE-MÜLLER, P.; FAJRELDIN, V.; CORONADO, V. L.; LÓPEZ-CONTRERAS, N.; VALENZUELA, M. T. Necesidades, creencias y prácticas en salud oral de padres y cuidadores de preescolares: Un enfoque cualitativo. Int. J. Odontostomat., 15(4):888-897, 2021.

mente 3 veces más probabilidades de tener daño por caries que los de NSE alto a los 2 años y 4 veces más probabilidades a los 4 años (Ministerio de Salud Chile, 2012). Avanzando en el ciclo vital, los/as niños/as chilenos/as de 12 años que se encuentran libres de caries sólo alcanza un $37,5 \%$, disminuyendo a un grave $0,8 \%$ en la población adulta de entre 35 y 44 años (Gamonal, 1996; Badenier, 2005; Soto \& Tapia, 2007a; Fresno et al.). Dicho esto, las políticas públicas en el país parecen no estar siendo del todo efectivas en el control del problema sugiriendo que, entre otros aspectos, es necesario reorientar los mensajes con los que se llega tanto a nivel individual como poblacional, a fin de establecer una comunicación efectiva que permita operar un cambio en las conductas en salud favorable a la disminución de estas tasas.

Al centrar el análisis en los datos publicados a nivel nacional en torno a las inequidades encontradas según ubicación geográfica, es posible constatar que mientras el 32,3\% de los niños de 6 años de áreas urbanas de nuestro país están libres de caries, sólo un $15,3 \%$ deellos lo está en las zonas rurales (Soto \& Tapia, 2007b; Fresno et al.). En esta misma lógica se observa que, de acuerdo a los resultados en relación a daño por caries, la historia de caries en niños de 6 años de localidades urbanas es de 3,44 dientes afectados, mientras que en localidades rurales aumenta a 5,04 (Soto \& Tapia, 2007a).

Esta disparidad en la producción social de la enfermedad responde a un mecanismo que involucra tanto el contexto económico como el sociocultural. De esta manera el nivel socioeconómico, como determinante estructural, expone diferencialmente a los individuos a recursos, además de definir su trayectoria en salud y condicionar un grado de vulnerabilidad en salud que es directamente proporcional a los niveles de exposición a los que haya estado sometido un individuo a lo largo de su ciclo vital (Vega, 2001; Baelum, 2011).

Por otro lado, el Modelo de Creencias en Salud (MCS), propuesto como una forma de explicar la relación existente entre las variables cognitivas y la adopción de conductas en salud, se ha adaptado para entender las variables cognitivas de los padres como un predictor para las prácticas en salud que desarrollan en relación a sus hijos (Rosenstock, 1974; Becker et al., 1977; Bush \& lannotti, 1990). De acuerdo a este modelo la adopción de determinada conducta en salud se asocia a 4 factores cognitivos (percepción de vulnerabilidad, percepción de severidad, percepción de barreras para la adherencia y percepción de la eficacia de la con- ducta en salud propiamente tal), capaces de influenciar el desarrollo de conductas asociadas a salud luego de ser "activadas" por estrategias tales como la educación o consejería otorgada por profesionales de la salud en torno a un tema determinado (Steele et al., 2001).

Ante este escenario, la Promoción de Salud se instala como una estrategia que permite afrontar la situación sanitaria actual como un catalizador de mejoras en términos de equidad en salud a nivel nacional en tanto contribuye a disminuir las brechas existentes entre las poblaciones urbana y rural (Soto \& Tapia 2007a).

Con el fin de disminuir la morbilidad bucal y mejorar los escasos resultados obtenidos entre los años 1992 y 2007, es que el estado de Chile ha desplegado variados programas de salud oral cuyo enfoque preventivo se orienta principalmente hacia la población infantil. Es así como en 2012 surge el Programa preventivo de Salud Bucal en población preescolar en Atención Primaria en Salud (APS), el cual en 2015 se denominó Programa Sembrando Sonrisas (Ministerio de Salud, 2019). Este programa se desarrolla como una estrategia de enfoque comunitario educativo que busca mantener y mejorar la salud bucal de la población parvularia (niños y niñas de entre dos y cinco años, 11 meses y 29 días) perteneciente a Junta Nacional de Jardines Infantiles (JUNJI), Fundación Integra y niveles de prekínder y kínder de escuelas municipalizadas y subvencionadas. Su componente educativo se aborda a través de talleres dirigidos a niños/as, educadoras y asistentes de párvulos y padres y apoderados, lo que constituye un complemento a la entrega de kits de higiene oral y aplicación bianual de barniz de flúor (Ministerio de Salud). Es un beneficio no postulable que permite acceder a un plan gratuito de salud bucal, para promover hábitos de higiene y alimentación, y la prevención de caries. Según datos disponibles del MINSAL, desde el año 2015 el programa se encuentra disponible en 326 comunas del país, y entre el año 2015 y 2016 ha beneficiado a más de 600.000 preescolares (Pepper et al., 2017). En tanto que evaluaciones recientes (Delpiano et al., 2019) han visto resultados positivos en torno a la prevención de las caries, posterior a la implementación del programa.

Este estudio buscó explorar los sistemas de creencias en torno a las necesidades en salud oral percibidas por padres y cuidadores/asde niños/as en edad preescolar que participaron en el pilotaje del Programa preventivo de Salud Bucal en población preescolar, resultados que fundamentaron la implementación de este programa a nivel nacional. 


\section{MATERIAL Y MÉTODO}

Se realizó un estudio cualitativo de tipo exploratorio-descriptivo, que relevó la percepción y experiencia de padres, madres y cuidadores de preescolares que asisten a establecimientos de educación preescolar dependientes de la JUNJI y Fundación Integra en las siete comunas participantes del pilotaje del Programa de Promoción y Prevención en Salud Bucal para población preescolar de Atención Primaria de Salud durante el año 2007. Entonces, las comunas urbanas y rurales fueron definidas a priori, las cuales correspondieron a Colchane, Illapel, Cerro Navia, Colina, Requinoa, Mulchén y Freire, localidades de Chile.

Para la selección de individuos se utilizó un muestreo intencionado en busca de aquellas personas que mejor podían responder a la pregunta de investigación, cuyos discursos posibilitaron conocer e interpretar el fenómeno estudiado.

La recolección y producción de información se llevó a cabo a través de entrevistas semiestructuradas como técnica de investigación. De acuerdo al contexto y la factibilidad de contar con un número suficiente de participantes, las entrevistas se conformaron de la siguiente manera:

- 4 Entrevistas Grupales

- 9 Focus Group

- 13 entrevistas individuales, de las cuales 5 fueron a apoderadas/os y 8 a educadoras.

Para el análisis, la información producida se abordó de acuerdo con los principios del análisis cualitativo de contenidos.
Respecto a los aspectos éticos, la participación de los individuos seleccionados para el estudio se realizó previa información de los objetivos del proyecto, origen del financiamiento y organización del estudio. Mediante consentimiento escrito se solicitó autorización voluntaria para la grabación de las entrevistas, garantizando la confidencialidad y anonimato de sus opiniones, suprimiendo las referencias que pudieran identificar a los entrevistados. Como una medida de protección de la privacidad de los entrevistados, el personal de los Jardines Infantiles no estuvo presente en ningún momento de la recolección de datos con las/os apoderadas/os.

\section{RESULTADOS}

El siguiente análisis identifica las asociaciones simbólicas emanadas desde el discurso de los entrevistados en torno a la salud oral, observándose las percepciones de los sujetos sobre el proceso de salud-enfermedad-atención.

A continuación, se presentan los elementos discursivos más relevantes asociados a los sistemas de creencias en salud oral por parte de padres y cuidadores de niños y niñas preescolares que fueron identificados en el transcurso de las distintas instancias de este estudio. Los resultados se presentan organizados en cuatro dimensiones temáticas elaboradas a fin de facilitar la presentación de los hallazgos.

1. Problemas y necesidades en salud de los y las preescolares. De acuerdo a lo expresado por los/as

Tabla I. Citas textuales en relación a la dimensión: "Problemas y Necesidades en salud de los y las Preescolares"

\begin{tabular}{|c|c|c|}
\hline $\mathrm{N}^{\circ}$ Cita & Transcripción & Fuente \\
\hline 1.1 & $\begin{array}{l}\text { "Yo creo que eso al menos en } m i \text { caso es más el resfriado que los } \\
\text { dientes, los dientes por ahora no me preocupan mucho, pero es súper } \\
\text { fácil que se resfríen y cuesta para sanarlo..." }\end{array}$ & Apoderada Jardín Infantil, Freire \\
\hline 1.2 & $\begin{array}{l}\text { "Una semana, dos semanas, un mes. Usted va a p edir la hora pa } \\
\text { cualquier cosa, pa pasar al doctor si te pueden dar la hora o pierdes la } \\
\text { hora... tienes que volver a sacar la hora y la pueden atender a las dos o } \\
\text { tres semanas después." }\end{array}$ & Apoderada Escuela, Freire \\
\hline 1.3 & $\begin{array}{l}\text { "...Y tener más capacidad para salud también dental igual...si es el colmo } \\
\text { que uno saque hora y te den para un mes más, y el niño está enfermo de } \\
\text { una muela..." }\end{array}$ & Apoderada Jardín Infantil, Colina \\
\hline 1.4 & $\begin{array}{l}\text { "Por ejemplo yo vengo de allá, me echo, cuando estoy con las pilas me } \\
\text { echo la hora justita. Cuando estoy con flojera, hora y media." }\end{array}$ & Apoderada Escuela, Freire \\
\hline
\end{tabular}


entrevistados/as es posible observar que apoderadas/ os no identifican los problemas relativos a salud oral como parte de las afecciones de salud que les resultan más relevantes y urgentes de resolver; encontrándose en primer término cuestiones relacionadas con enfermedades de las vías respiratorias y gastrointestinales (Tabla I, CITAS 1.1, 1.2).

Por otro lado, entre los problemas que se expresan con mayor ímpetu transversalmente en el discurso dicen relación con la falta de horas disponibles en el Sistema Público y lo poco oportuno que resulta el tratamiento recibido (Tabla I, CITA 1.3). Adicionalmente, los entrevistados residentes en las zonas rurales relevan la distancia de su domicilio al centro asistencial como una importante dificultad para el acceso a la atención (Tabla I, CITA 1.4).
2. Hábitos relacionados con Salud. En relación con los hábitos saludables en las zonas rurales, prácticas tales como la higiene corporal se ven limitadas y condicionadas a las condiciones climáticas, esto debido a que las viviendas no cuentan con baño al interior de la casa (Tabla II, CITA 2.1). Bajo esas condiciones la frecuencia del baño está relacionada con la época del año, cuestión que eventualmente podría repercutir en el hábito de higiene bucal.

De acuerdo al discurso de los entrevistados, el adecuado estado de salud de un/a niño/a se asocia al desarrollo de todas sus potencialidades funcionales (alimentarse bien, jugar, dormir bien, comunicarse, etc.), así como también al "cumplimiento" de actividades que dicta el sistema oficial de salud (Tabla II, CITA 2.2).

Tabla II. Citas textuales en relación a la dimensión:" Hábitos relacionados con Salud".

\begin{tabular}{|c|c|c|}
\hline $\mathrm{N}^{\circ}$ Cita & Transcripción & Fuente \\
\hline 2.1 & $\begin{array}{l}\text { "Igual que esas películas antiguas, con un lavatorio y un jarro. Así } \\
\text { nos bañamos en el campo (...) hervimos en una tetera grande." }\end{array}$ & Entrevistadas Escuela, Freire \\
\hline 2.2 & $\begin{array}{l}\text { "Mi niño es súper activo, que quiere jugar a la pelota...con } \\
\text { agua...para mí es un niño sano, le tengo todas las vacunas al día, a } \\
\text { control..." }\end{array}$ & Apoderadas Jardines Infantil, Colina \\
\hline 2.3 & $\begin{array}{l}\text { "Mi hijo se lava harto los dientes, yo nunca lo he llevado al } \\
\text { dentista...lo que he tratado de ver...no me he dado cuenta que } \\
\text { tenga caries, de hecho por lo mismo porque a él le gusta lavarse con } \\
\text { pasta y se los lava él, después se los vuelvo a lavar yo..." }\end{array}$ & Apoderada Jardín Infantil, Colina \\
\hline 2.4 & $\begin{array}{l}\text { "...a ella de primera cuando recién le habian salido, con un pañito. } \\
\text { Le compré un cepillo y claro le paso el cepillo un poco pero ella me } \\
\text { lo quita y después igual le paso un paño..." }\end{array}$ & Apoderada Jardín Infantil, Freire \\
\hline 2.5 & $\begin{array}{l}\text { "Nos pidieron pasta y cepillo. Yo al menos mandé y un día pregunté } \\
\text { porque llegaba igual como la había echado....la tía en el día le lava } \\
\text { porque cuando cambian la tía en la extensión horaria no tiene } \\
\text { tiempo..." }\end{array}$ & Apoderada Jardín Infantil, Freire \\
\hline 2.6 & $\begin{array}{l}\text { “...yo a mi hijo le enseño...como la doctora me enseñó a mí a } \\
\text { lavarme los dientes...suponga esta es la encía de aquí de la encía } \\
\text { pa arriba tenemos que sacar toda la mugre que queda de la encía, } \\
\text { porque cuando queda con mugre la encía ahí se pega la caries, eso } \\
\text { le enseño yo a mi hijo. Y mi hijo me dice pero mamá ya estoy } \\
\text { choreado de lavarme los dientes...No, lávate los dientes bien. Y la } \\
\text { doctora siempre me regala enjuague y él me dice ya, yo me voy a } \\
\text { enjuagar. Le digo no...., tienes que lavártelos primero y después } \\
\text { enjuagártelo...” }\end{array}$ & Apoderada Jardín Infantil, Cerro Navia \\
\hline 2.7 & $\begin{array}{l}\text { "Sí, privarle de golosinas, hay niños que comen demasiadas } \\
\text { golosinas y por eso le salen caries. Y tenerles el horario igual de } \\
\text { comidas" }\end{array}$ & Apoderada Jardín Infantil, Freire \\
\hline 2.8 & $\begin{array}{l}\text { "...en la noche le lava los dientes después de la comida, pero la } \\
\text { última en la noche es la mamadera y se acuesta con eso. Lo ideal es } \\
\text { darle primero la leche, lavarle los dientes y nada más...para que eso } \\
\text { trabaje bien en su estómago y baje bien esa cosita y no le vuelva a } \\
\text { la parte bucal...eso pienso yo" }\end{array}$ & Apoderada Jardín Infantil, Freire \\
\hline
\end{tabular}


En contraste con lo anterior, en lo que respecta a la salud oral el cepillado dental es identificado como el principal hábito protector contra la caries dental; siendo entonces una estrategia de auto control que minimiza la importancia de la visita al odontólogo (Tabla II, CITA 2.3). Sin embargo, las dificultades en la instauración del hábito de cepillado se vinculan a una falta de "voluntad" por parte de los niños (Tabla II, CITA 2.4). En esta misma línea, la mayoría de los entrevistados relata realizar el cepillado de dientes dos veces al día (en la mañana y en la noche), siendo responsabilidad del jardín la realización del cepillado a lo largo del día. No obstante a ello, gran parte de los apoderados refieren que las educadoras no realizan el cepillado por falta de tiempo (Tabla II, CITA 2.5). En torno a la lógica de "recordar" al niño que debe cepillarse, el discurso ha sido reiterativo en referir el rol de la madre y su responsabilidad como agente socializador de la versión oficial entregada por el profesional odontólogo, sin embargo y de acuerdo a lo evidenciado por los entrevistados, este discurso se adapta a la propia lógica de la madre (Tabla II, CITA 2.6).

Por otro lado, aun cuando gran parte de los entrevistados/as son capaces de identificar buenas prácticas de manera conceptual, no las describen como parte de su cotidianeidad; así es como aun cuando asocian el consumo de verduras y lácteos con la salud oral, su práctica no es habitual dada la falta de recursos en zonas empobrecidas del sector rural. De igual modo, respecto al consumo de alimentos, se identifica claramente en el discurso que la alta frecuencia de consumo de golosinas es riesgoso para la salud oral, mientras que la generación de horarios actúa como factor protector (Tabla II, CITA 2.7).

Respecto a la prevención de patologías orales, el consumo de alimentos en la noche es aludido por las mujeres jóvenes como un hábito que debe evitarse; reflejándose la influencia de las indicaciones oficiales recibidas en el contexto asistencial. Aún así, pese a que el uso de biberón nocturno es un elemento identificado como un potencial factor productor de caries, corresponde a una conducta frecuente y validada (Tabla II, CITA 2.8).

\section{Rol que ejercen cuidadores/as en torno a la sa-} lud oral de preescolares. Según se expresa de manera recurrente en el discurso de los entrevistados, la visita al odontólogo en los preescolares no se realiza con fines preventivos, sino que se posterga has- ta la existencia de la patología. Así, según los padres, el rol de los dentistas está asociado a la reparación del daño existente, lo cual implica la realización de acciones invasivas y muchas veces dolorosas (Tabla III, CITA 3.1 y 3.2).

Paradojalmente, se constata que en algunos casos el discurso de los apoderados tiende a asignar la responsabilidad del estado de salud buco-dental de los niños al profesional odontólogo, lo que resulta contradictorio frente a lo poco oportuna que ha sido la primera consulta (Tabla III, CITA 3.3).

Así mismo, junto con aludir al rol del odontólogo desde la perspectiva asistencial como un prestador de atención terapéutica, los entrevistados analizan el rol que "debiese" tener el odontólogo, desde lo cual emerge la falta de inducción y educación en salud oral que éste realiza para con los usuarios y expresan su voluntad de querer recibir esta prestación en un lenguaje que resulte adecuado y comprensible para ellos (Tabla III, CITAS 3.4 y 3.5).

En otro orden de cosas, es posible apreciar en el discurso que los participantes no asumen un rol protagónico en la adquisición de hábitos ni en la prevención de caries dental de sus hijos e hijas, de manera que las caries son explicadas por factores externos, tales como la "mala calidad" de los dientes, la herencia o la falta de atención profesional (Tabla III CITA 3.6 y 3.7 ).

Desde otra perspectiva y según lo expresan los padres, madres y apoderados, la adopción de medidas de prevención de la caries dental se asocia con un rol de transmisión -o superación- de los estigmas sociales que tradicionalmente caracterizan a una población empobrecida, tales como un aspecto físico que el propio sujeto considera denigrante (Tabla III, CITA 3.8).

Igualmente es posible identificar otros agentes socializadores de contenidos de salud oral a la vez que replicadores de los prejuicios y expectativas sociales, siendo los medios de comunicación mencionados en reiteradas ocasiones, puesto que en ocasiones promueven el consumo de elementos tales como dulces y golosinas, considerados dañinos en cuanto a la producción de caries (Tabla III, CITA 3.9).

4. Imaginario de la Salud y Enfermedad Bucal. Entre las problemáticas de salud oral más mencionadas, surgen la caries (como un factor asociado a la des- 
OLAVE-MÜLLER, P.; FAJRELDIN, V.; CORONADO, V. L.; LÓPEZ-CONTRERAS, N.; VALENZUELA, M. T. Necesidades, creencias y prácticas en salud oral de padres y cuidadores de preescolares: Un enfoque cualitativo. Int. J. Odontostomat., 15(4):888-897, 2021.

Tabla III. Citas textuales en relación a la dimensión: "Rol que ejercen cuidadores/as en torno a la salud oral de preescolares"

\begin{tabular}{|c|c|c|}
\hline $\begin{array}{l}\mathrm{N}^{\circ} \\
\text { Cita }\end{array}$ & Transcripción & Fuente \\
\hline 3.1 & $\begin{array}{l}\text { "...en los (dientes) principales tenía una carie chiquitita. El dentista le } \\
\text { puso..., la pasó la máquina sin ponerle anestesia...lo volví a llevar y le dije: } \\
\text { No, si le hace algo se lo hace con anestesia...la máquina va a ser constante } \\
\text { dolor ahi porque se supone que tiene vivo los dientes. El más grande le } \\
\text { tiene miedo ir al dentista, es un trauma ir al dentista." }\end{array}$ & $\begin{array}{l}\text { Apoderada Jardín Infantil, } \\
\text { Colina }\end{array}$ \\
\hline 3.2 & $\begin{array}{l}\text { "No lo he llevado al dentista todavía porque...no quiero que lo hagan sufrir... } \\
\text { no, le duele mucho. Igual yo le limpio bien." }\end{array}$ & $\begin{array}{l}\text { Apoderada Jardín Infantil, } \\
\text { Colina }\end{array}$ \\
\hline 3.3 & "Que estén sanos los dientes, ese rol juega él" & $\begin{array}{l}\text { Apoderada Jardín Infantil, } \\
\text { Freire }\end{array}$ \\
\hline 3.4 & $\begin{array}{l}\text { "Que use otro tipo de palabras más de entrada, que somos dueñas de casa } \\
\text { pero de repente el roce no va a ser nunca un roce alto, o sea por último un }\end{array}$ & $\begin{array}{l}\text { Apoderada Jardín Infantil, } \\
\text { Illapel }\end{array}$ \\
\hline
\end{tabular}

roce de nosotras de la casa, que pasa esto, que el diente esta picao...esa son las maneras..."

3.5 "...ella a mí en ningún momento me dice mire tiene que asi lavarse los dientes o que pasta tiene que usar, no. Solamente ella hace el trabajo que tiene que hacer... y nada más."

3.6 "No se si estaré en lo correcto, pero el tema de las caries es un tema de defensas, porque hay niños que le salen los dientes a muy temprana edad, entonces así como salen más temprano las caries llegan más temprano todavía y algunos que son más tardíos se demoran a veces años en que tengan caries cuando comen dulces. Entonces yo creo que también es un tema genético, puedes ser, no tengo conocimiento de ello."

3.7 "... si po, si lo atienden obvio que estarian sanitos, pero si uno lo lleva y no quieren atenderlo o si se preocuparán más y salieran, bueno, no habría tanto problema en los niños, las muelas tan carias."

3.8 "...yo creo que por eso hay que cuidarle la dentadura a los niños porque cuando lleguen a adultos tengan su buena dentadura, se vean bien, tengan buena presencia para trabajar. Si no tiene los dientes una se ve fea...uno

Apoderada Jardín Infantil, Illapel

Apoderada Jardín Infantil, Freire tiene que aprender a cuidarle los dientes a los hijos pa que no les pase lo mismo..."

3.9 "...todo lo que es comida chatarra, los dulces, las golosinas, éstas...cereales. Todo eso los niños están comiendo prácticamente todos los días o sea esa es una lucha constante aquí en el jardín para que no traigan esas cosas. El uso del chicle ponte tú. Yo llevo diez años en este jardín infantil, diez años que estoy hablando acerca del chicle en los niños y todavía tengo niños comiendo chicle..."

Apoderada Jardín Infantil, Freire

Apoderada Jardín Infantil, Cerro Navia

Educadores de Párvulos Jardín Infantil, Illapel

trucción del diente), las anomalías dentomaxilares (identificadas principalmente como malposicones) y la coloración "amarilla" de los dientes (relevada desde el punto de vista estético). Sin embargo, no es posible identificar en el discurso un análisis en profundidad respecto a la etiología de estas patologías, limitándose los hallazgos sólo a la descripción de su frecuencia.
Por otro lado, y como consecuencia de la valoración hacia la mantención de los dientes en boca, la extracción dentaria no es un tratamiento deseado por los padres y apoderados tanto para las/os niña/os como para ellos mismos, cuestión que contribuye a la falta de confianza en el sistema (Tabla IV, CITA 4.1 y 4.2). 
OLAVE-MÜLLER, P.; FAJRELDIN, V.; CORONADO, V. L.; LÓPEZ-CONTRERAS, N.; VALENZUELA, M. T. Necesidades, creencias y prácticas en salud oral de padres y cuidadores de preescolares: Un enfoque cualitativo. Int. J. Odontostomat., 15(4):888-897, 2021.

Tabla IV. Citas textuales en relación a la dimensión: "Construcción Social de la Enfermedad”

\begin{tabular}{|c|c|c|}
\hline $\mathrm{N}^{\circ}$ Cita & Transcripción & Fuente \\
\hline 4.1 & $\begin{array}{l}\text { "...Lo más fácil pa ellos es sacarla en vez de taparla, lo más rápido... por } \\
\text { eso uno le da miedo ir...” }\end{array}$ & $\begin{array}{l}\text { Apoderada Jardín Infantil, } \\
\text { Colina }\end{array}$ \\
\hline 4.2 & $\begin{array}{l}\text { "Eso, cuando una era niña los papas los llevaban al dentista: chao y } \\
\text { fuera. Y resulta que acá uno no quiere que los niños pierdan dientes." }\end{array}$ & $\begin{array}{l}\text { Apoderada Jardín Infantil, } \\
\text { Colina }\end{array}$ \\
\hline 4.3 & $\begin{array}{l}\text { "Uno se da cuenta de una caries cuando ya tiene el hoyo, pero hay veces } \\
\text { que tiene caries blancas que también uno no se da cuenta..." }\end{array}$ & $\begin{array}{l}\text { Apoderada Jardín Infantil, } \\
\text { Colina }\end{array}$ \\
\hline 4.4 & $\begin{array}{l}\text { "Por lo que a mi me han dicho..., yo tengo entendido que después de } \\
\text { comer y antes de acostarse deben lavarse los dientes, no comer, lavarse } \\
\text { los dientes después de una mamadera y de ir a dormir, porque ahí se } \\
\text { queda toda la mugre en la boca, e igual llegan los bichitos y las caries. } \\
\text { Los dulces igual bien restringidos, bien a lo lejos, y trato de darle...ella es } \\
\text { buena para la fruta, le encanta. Yo sé que la fruta tiene más protección } \\
\text { para los dientes y también, le gusta mucho las ensaladas." }\end{array}$ & $\begin{array}{l}\text { Apoderada Jardín Infantil } \\
\text { Cerro Navia }\end{array}$ \\
\hline 4.5 & $\begin{array}{l}\text { "Tiene que ser por la comida, más frutita, más calcio en los dientes. Están } \\
\text { muy débiles los dientes hoy día." }\end{array}$ & $\begin{array}{l}\text { Apoderada Jardín Infantil, } \\
\text { Freire }\end{array}$ \\
\hline
\end{tabular}

En el mismo sentido, el discurso refiere a diversos signos externos que se consideran indicadores de caries, pero sin aludir a qué es la caries conceptualmente. De esta manera, se alude al cambio de color de los dientes como indicador de in-sanidad, tema que debe comprenderse en un contexto de fuerte propaganda mediática donde lo valioso y bello es la "blancura"de la sonrisa.

Así, la severidad de la caries se asocia a la coloración, de forma que el color negro se encuentra en aquellos casos donde existe destrucción evidente del diente, y el color blanco en aquellos procesos cariosos incipientes que no pueden ser observados por los apoderados, sino sólo por el profesional o equipo de salud odontológico. El discurso de "caries blancas" y "caries negras" refleja entonces el que los apoderados reconocen a la caries como un proceso progresivo, diferenciándose del discurso que plantea la aparición de caries y la destrucción dentaria como un fenómeno espontáneo. Este último aparece como explicación ante la imposibilidad de no haber intervenido con anterioridad y de la creencia que el desarrollo de caries es el resultado de eventos no manejables por los apoderados (Tabla IV, CITA 4.3).

La caries, como entidad nosológica, no queda suficientemente descrita por el conjunto del discurso emanado de la estrategia metodológica utilizada. Ello contrasta con la profusión del discurso en torno a factores que la producen, pero refleja a su vez un imaginario pobre y vago sobre qué es la caries y la ausencia de la idea de producción multifactorial (Tabla IV, CITA 4.4).
Por otro lado, pese a que los apoderados logran identificar ciertos alimentos como productores de caries tales como alimentos con alto contenido de azúcar, no incluye los productos salados, ni tampoco aquellos alimentos que aun conteniendo azúcar son catalogados como saludables tales como yogurt- fruta, verduras, lácteos y sus derivados, siendo incluso descritos como protectores para el desarrollo de caries. El calcio presente en los productos lácteos es el elemento identificado como protector de la salud de los dientes, pero sin profundizar en la explicación (Tabla IV, CITA 4.5).

\section{DISCUSIÓN}

En términos generales, la literatura reporta que los niños provenientes de familias de escasos recursos tienen menos posibilidades de acceder a atención integral en salud oral y, por consiguiente, presentan mayores niveles de daño agudo en contraste con niños que provienen de familias con mayores ingresos (Mouradian et al., 2000; Edelstein, 2002; Kelly et al., 2005). En suma, los niños pertenecientes a minorías étnicas o raciales, provenientes de familias numerosas y/o cuyos padres poseen un bajo nivel de escolaridad, tienen un menor acceso a los servicios de atención dental (Edelstein ; Kelly et al.). Todos estos elementos constituyen barreras de acceso que pueden explicar la falta de adherencia al sistema de salud (Steele et al.). En el caso de los resultados del estudio se vio reflejado en los discursos de las personas residentes en zonas rurales, los cuales mencionaron más barreras para mantener una salud oral óptima. 
OLAVE-MÜLLER, P.; FAJRELDIN, V.; CORONADO, V. L.; LÓPEZ-CONTRERAS, N.; VALENZUELA, M. T. Necesidades, creencias y prácticas en salud oral de padres y cuidadores de preescolares: Un enfoque cualitativo. Int. J. Odontostomat., 15(4):888-897, 2021.

Por otro lado, el modelo conceptual de FisherOwens, presenta una organización multinivel de los factores que inciden en la salud oral de los niños tanto a nivel individual como familiar y comunitario (FisherOwens et al., 2007). En el nivel familiar la influencia es ejercida principalmente por los/as cuidadores con quienes los niños pasan la mayor parte del tiempo, siendo posible afirmar que las conductas en salud adoptadas por los niños son influenciadas, ya sea manera directa o indirecta, por el conocimiento, actitudes, creencias y prácticas de los padres y cuidadores (Blinkhorn, 1991; Naidu et al., 2012). En esta línea, diversos estudios refieren que los padres y cuidadores que subutilizan el sistema de salud dental aseguran ser un mal ejemplo para sus hijos, poseer un escaso nivel de conocimientos en salud dental y atribuir a terceros (las escuelas y los propios odontólogos) la responsabilidad de transmitir información referente a salud oral a los niños (Kelly et al.). Respecto a este último punto y tal como se puede constatar en los resultados de este estudio, el nivel de responsabilidad asumido por los padres en torno a la atención odontológica preventiva se encuentra fuertemente influenciado por su propio sistema de creencias y normas, las cuales pueden ser modificadas mediante programas de educación en salud (Galanos et al. 1994; Green \& Kelly, 2004; Steadman \& Rutter, 2004). En esta misma línea, la literatura reporta que los padres y cuidadores que adoptan un rol activo en el cuidado de la salud oral de sus hijos están al tanto de los cuidados preventivos que deben adoptar, así como también de las consecuencias a largo plazo que las morbilidades orales tienen en la dentición de los preescolares (Kelly et al.).

En otro orden de cosas, la idea de que la dentición decidua es temporal y, por lo tanto, no relevante, se encuentra bastante asentada entre las/os cuidadores, siendo un pensamiento común que ha podido constatarse en otros estudios similares (Hilton et al., 2007; Obeng, 2008; Mofidi et al., 2009; Naidu et al.). Esta falta de claridad en cuanto a su rol e importancia en el desarrollo de la dentición permanente genera confusiones respecto a cuando resulta oportuno realizar la primera visita al odontólogo siendo, según lo expresado por los/as entrevistados/ as, postergada hasta la aparición del daño, mas no con fines preventivos, aún cuando existe evidencia que muestra que la atención temprana aumenta las posibilidades de no presentar caries durante la infancia (al Ghanim et al., 1998; Naidu et al.).

En relación a los hábitos alimenticios de los niños y niñas, las/os entrevistadas/os parecen estar conscientes de la importancia de limitar el consumo de productos altos en azúcares con el fin de prevenir la caries dental, haciendo énfasis en potenciar el consumo de alimentos que asocian a una buena salud como la leche, yogurt y fruta. No obstante, existen factores culturales asociados al acceso que tienen los niños y niñas a alimentos altamente azucarados, los cuales son asociados a refuerzos positivos y muestras de cariño por parte de abuelas/os, familiares y vecinos/as (Hunter et al., 1997; Naidu et al.).

Como elemento importante, cabe destacar que las conductas en salud oral adoptadas por parte de madres y padres, a la vez de su nivel de involucramiento que asumen respecto a la salud de sus hijos, están fuertemente asociados a la "Autoeficacia", entendida como la convicción de un individuo respecto a su propia capacidad de adoptar una conducta requerida para lograr determinado objetivo y llevar a cabo con éxito una tarea específica (Bandura, 1977; Naidu et al.). Con respecto a este punto, estudios demuestran que la autoeficacia percibida por madres y padres fue el mayor predictor de éxito en el establecimiento de hábitos de higiene oral y el control en la ingesta de alimentos azucarados, destacándose que la autoeficacia percibida era directamente proporcional al nivel de conocimientos que poseían madres y padres, lo que releva las estrategias de promoción de la salud con enfoque educativo, como lo es el programa de salud bucal nacional "Sembrando Sonrisas" (Adair et al., 2004; Finlayson et al., 2007; Naidu et al.).

Estudios recientes que analizan resultados del programa han evidenciado cambios en relación a nuestros hallazgos. Es así como en el estudio realizado en la comuna de Puente Alto (Chile) el año 2020, evidenció que las madres tienen mayor protagonismo en el cuidado de la salud oral de los niños, así como la percepción de mayor acceso a atención dental por parte de los centros de atención primaria. Por otro lado, ese estudio coincide con la valoración que implica la estética dental de los niños y niñas en la evaluación de la calidad de la salud oral (Humeres-Flores et al., 2020).

Como limitación de este estudio encontramos que la información se podría haber ampliado considerando otros grupos como los mismos niños o los odontólogos de los centros de salud, sin embargo, la información que se recogió fue útil para generar el programa que actualmente se sigue ejecutando. Como fortaleza, este estudio fue realizado en dife- 
rentes zonas geográficas y su metodología permitió recoger un amplio abanico de percepciones en torno a la vida de las madres y educadoras en relación al cuidado de la salud oral.

En conclusión, es importante realizar este tipo de estudios, para complementar los resultados de corte más cuantitativos que va obteniendo el programa. Entender qué hay detrás de los indicadores, a través de las creencias en salud de madres, padres y cuidadores/as, potencia los insumos para generar mayores acciones en relación a la prevención de enfermedades de salud oral tanto en los niños y niñas, como a lo largo del ciclo vital.

OLAVE-MÜLLER, P.; FAJRELDIN, V.; CORONADO, V. L.; LÓPEZ-CONTRERAS, N.; VALENZUELA, M. T. Needs, beliefs and practices in oral health of preschoolers' parents and caregivers: A qualitative scope. Int. J. Odontostomat., 15(4):888-897, 2021.

ABSTRACT: Almost ten years after the implementation of the successful public oral health promotion program for preschoolers in Chile, we present the previous study that collected the vision and meanings for parents about oral health issues of their preschool children in urban and rural Chile in seven different communities. The high prevalence of dental caries and their consequences throughout the lifetime in Chile has made health promotion a relevant strategy that allows facing the current health situation by implementing prevention-oriented programs focused on the pre-school level population, like the "Sembrando Sonrisas" program whose educational component focuses on parents and caregivers. In this line, it is worth mentioning the Health Beliefs Model (HBM), which acts as a predictor for the health practices that parents develop concerning their children, which can be activated and or modified by educational strategies or counseling provided by health professionals. This study seeks to explore, through qualitative methodology, the belief system around the oral health needs perceived by parents and caregivers of preschool children who participated in the testing of the Preventive Oral Health Program in pre-school level population in 7 counties of the country during 2007 . The results obtained showcase that parents and guardians assign oral health care to third parties (dentists, pre-school educators) while reporting a lack of understandable information so that they can replicate practices in their children. On the other hand, postponing the visit to the dentist is justified due to previous bad experiences and lack of access to dental care services. However, dental health is relevant from an aesthetic point of view and as an element to overcome social prejudice.

KEY WORDS: preschool children, health behaviors, oral health

\section{REFERENCIAS BIBLIOGRÁFICAS}

Adair, P. M.; Pine, C. M.; Burnside, G.; Nicoll, A. D; Gillet, A.; Anwar, S.; Broukal, Z.; Chestnutt, I. G.; Declerck, D.; Xi Ping, F.; et al. Familial and cultural perceptions and beliefs of oral hygiene and dietary practices among ethnically and socio-economically diverse groups. Community Dent. Health, 21(1 Suppl.):102-11, 2004.

al Ghanim, N. A.; Adenubi, J. O.; Wyne, A. A. \& Khan, N. B. Caries prediction model in preschool children in Riyadh, Saudi Arabia. Int. J. Paediatr. Dent., 8(2):115-22, 1998.

Badenier, O.; Cueto, A. \& Moya, R. Estudio de Prevalencia de las enfermedades bucodentales y necesidades de tratamiento en la V Región. FONIS SA05I20044, 2005.

Baelum, V. Dentistry and population approaches for preventing dental diseases. J. Dent., 39 Suppl. 2:S9-19, 2011.

Bandura, A. Toward a unifying theory of behavioural change. Psychol. Rev., 84:191-215, 1977.

Becker, M. H.; Maiman, L. A.; Kirscht, J. P.; Haefner, D. P. \& Drachman, R. H. The Health Belief Model and prediction of dietary compliance: a field experiment. J. Health Soc. Behav., 18(4):34866, 1977

Blinkhorn, A. Promoting dietary changes in order to control dental caries. Dent. Health, 29:3-6, 1991.

Bush, P. J. \& lannotti, R. J. A children's health belief model. Med. Care, 28(1):69-86, 1990.

Delpiano, D.; Moya, P. \& Vidal, C, Estrategia preventiva para el control de la caries dental en preescolares de establecimientos educacionales municipalizados. Medwave, 19(S1):SP107, 2019.

Edelstein, B. L. Disparities in oral health and access to care: findings of national surveys. Ambul. Pediatr., 2(2 Suppl.):141-7, 2002.

Finlayson, T; Siefert, K; Ismail, A; Sohn, W. Maternal self-efficacy and 1-5-year-old children's brushing habits. Community Dent. Oral Epidemiol., 35(4):272-81, 2007.

Fisher-Owens, S. A.; Gansky, S. A.; Platt, L. J.; Weintraub, J. A.; Soobader, M. J.; Bramlett, M. D. \& Newacheck, P. W. Influences on children's oral health: a conceptual model. Pediatrics, 120(3):e510-20, 2007.

Fresno, C.; Jeldes, G.; Estay, J. \& Martin, J. Prevalencia, severidad de caries dental y necesidad de tratamiento restaurador en escolares de 6 a 12 Años de la Provincia de Santiago, Región Metropolitana. Rev. Clin. Periodoncia Implantol. Rehabil. Oral., 12(2):81-6, 2019.

Galanos, A. N.; Strauss, R. P. \& Pieper, C. F. Sociodemographic correlates of health beliefs among black and white community dwelling elderly individuals. Int. J. Aging Hum. Dev., 38(4):33950, 1994

Gamonal, J. Prevalencia de enfermedades periodontales y de caries dental en la población de 35-44 y de 65 a 74 años de nivel socioeconómico bajo y medio-bajo de la provincia de Santiago, Región Metropolitana, y determinación de los recursos humanos necesarios para su tratamiento. Rev. Fac. Odontol. Univ. Chile, 14(1):56-7, 1996.

Green, P. M. \& Kelly, B. A. Colorectal cancer knowledge, perceptions, and behaviors in African Americans. Cancer Nurs., 27(3):20615,2004

Hilton, I. V.; Stephen, S.; Barker, J. C. \& Weintraub, J. A. Cultural factors and children's oral health care: a qualitative study of carers of young children. Community Dent. Oral Epidemiol., 35(6):42938,2007

Humeres-Flores, P.; Guzmán-Orellana, D.; Madrid-Canales, C.; Fredes-Ziliani, A. \& Mustakis-Truffello, A. Cuidado de la salud oral en la primera infancia: La perspectiva de sus madres-un estudio cualitativo. Int. J. Int. Dent., 13(2):62-6, 2020. 
OLAVE-MÜLLER, P.; FAJRELDIN, V.; CORONADO, V. L.; LÓPEZ-CONTRERAS, N.; VALENZUELA, M. T. Necesidades, creencias y prácticas en salud oral de padres y cuidadores de preescolares: Un enfoque cualitativo. Int. J. Odontostomat., 15(4):888-897, 2021.

Hunter, M. L.; Hood, C. A.; Hunter, B. \& Kingdon, A. Reported infant feeding, oral hygiene and dental attendance patterns in children aged 5 years and under referred for extraction of teeth under general anaesthesia. Int. J. Paediatr. Dent., 7(4):243-8, 1997.

Kelly, S. E.; Binkley, C. J.; Neace, W. P. \& Gale, B. S. Barriers to care-seeking for children's oral health among low-income caregivers. Am. J. Public Health, 95(8):1345-51, 2005.

Ministerio de Salud. Orientaciones Técnico Administrativas para Ejecución de Programa Sembrando Sonrisas 2019. Santiago de Chile, Ministerio de Salud, División de Atención Primaria, Unidad Odontológica, Gobierno de Chile, 2019.

Mofidi, M.; Zeldin, L. P. \& Rozier, R. G. Oral health of early head start children: a qualitative study of staff, parents, and pregnant women. Am. J. Public Health, 99(2):245-51, 2009.

Mouradian, W. E.; Wehr, E. \& Crall, J. J. Disparities in children's oral health and access to dental care. JAMA, 284(20):2625-31, 2000.

Naidu, R.; Nunn, J.; Forde, M. Oral healthcare of preschool children in Trinidad: a qualitative study of parents and caregivers. BMC Oral Health, 12:27, 2012.

Obeng, C. Dental care issues for African immigrant families of preschoolers. Early Child. Care Pract., 10:2, 2008.

Organización Mundial de la Salud (OMS). Salud Bucodental. Ginebra, Organización Mundial de la Salud (OMS), 2020. Disponible en: https://www.who.int/es/news-room/fact-sheets/detail/oralhealth

Pepper, S.; Arce, R.; Saint-Pierre, E. \& Vicencio, C. (Eds.). Informe Final De Evaluación Evaluación Programas Gubernamentales (EPG). Programa Sembrando Sonrisas. Santiago de Chile, Subsecretaría de Redes Asistenciales, Ministerio de Salud, Gobierno de Chile, 2017. Disponible en: dipres.gob.cl/597/articles163117_informe_final.pdf

Rosenstock, I. M. Historical origins of the health belief model. Health Educ. Monogr., 2(4):328-35, 1974.

Soto, Q. L. \& Tapia, C. R. (Eds.). Diagnóstico Nacional de Salud Bucal de los niños de 6 años. Santiago de Chile, Ministerio de Salud, Gobierno de Chile, 2007a. Disponible en: https:// diprece.minsal.cl/wrdprss_minsal/wp-content/uploads/2015/05/ Diagnostico-Nac-Salud-Bucal-ni \%C3 \%B1os-6-a \%C3 \%B1os_Chile-2007.pdf

Soto, Q. L. \& Tapia, C. R. (Eds.). Diagnóstico Nacional de Salud Bucal del Adolescente de 12 años y Evaluación del Grado de Cumplimiento de los Objetivos Sanitarios de Salud Bucal 20002010. Santiago de Chile, FONIS (MINSAL-CONICYT), Subsecretaría de Salud Pública, Universidad Mayor, Colgate/Palmolive, 2007b.

Steadman, L. \& Rutter, D. R. Belief importance and the theory of planned behaviour: comparing modal and ranked modal beliefs in predicting attendance at breast screening. $\mathrm{Br}$. J. Health Psychol., 9(Pt. 4):447-63, 2004.

Steele, R. G.; Anderson, B.; Rindel, B.; Dreyer, M. L.; Perrin, K.; Christensen, R.; Tyc, V. \& Flynn, P. M. Adherence to antiretroviral therapy among HIV-positive children: examination of the role of caregiver health beliefs. AIDS Care,13(5):617-29, 2001.

Vega, J. Enfermo de pobre: Las inequidades sociales y la salud en Chile. Rev. Univ. (Santiago), 73:43-56, 2001.
Dirección para correspondencia:

Paola Olave Müller

Universidad de La Frontera

Facultad de Medicina

Departamento de Salud Pública

Temuco

CHILE

E-mail: paola.olave@ufrontera.cl 